\title{
Rapid incorporation of omega-3 fatty acids into blood mononuclear cells
}

\author{
A. L. West, G. C. Burdge and P. C. Calder \\ Human Development and Health Academic Unit, Faculty of Medicine, University of Southampton, Southampton SO16
}

$6 Y D, U K$

The omega-3 fatty acids eicosapentaenoic acid (EPA) and docosahexaenoic acid (DHA) are known to influence inflammation and immunity $^{(1)}$. The effects seen have been related to increased incorporation of these fatty acids into the membranes of the cells involved ${ }^{(2)}$. This incorporation has been typically studied over a time course of weeks to months ${ }^{(3)}$, although recent studies reveal appearance of EPA and DHA in human blood mononuclear cells (MNCs) within a few days of increased intake ${ }^{(4)}$. Here we investigate whether there is an increased content of EPA and DHA in MNCs 6 hours following consumption of the fatty acids and whether the chemical formulation of the omega- 3 fatty acids matters.

Healthy male volunteers $(n$ 10) aged 18-40 y were recruited into a double blinded cross-over trial. Each recruit consumed EPA and DHA in the 5 different chemical forms ("natural" triacylglycerol (TG), reconstituted TG (rTG), enterically-coated reconstituted TG (rTG entericare), ethyl ester (EE), free fatty acid (FFA)) on 5 post-prandial study days each at least 2 weeks apart. The supplements were taken in random order. At each clinic visit the fasted recruits were cannulated, baseline blood samples collected, and a standard breakfast consumed along with the supplement. All supplements provided $1.1 \mathrm{~g}$ EPA and $0.4 \mathrm{~g}$ DHA. Blood samples were collected at baseline and at 6 hours. MNCs were isolated using a Histopaque-Ficoll gradient. Incorporation of EPA and DHA into MNCs was assessed by gas chromatography.

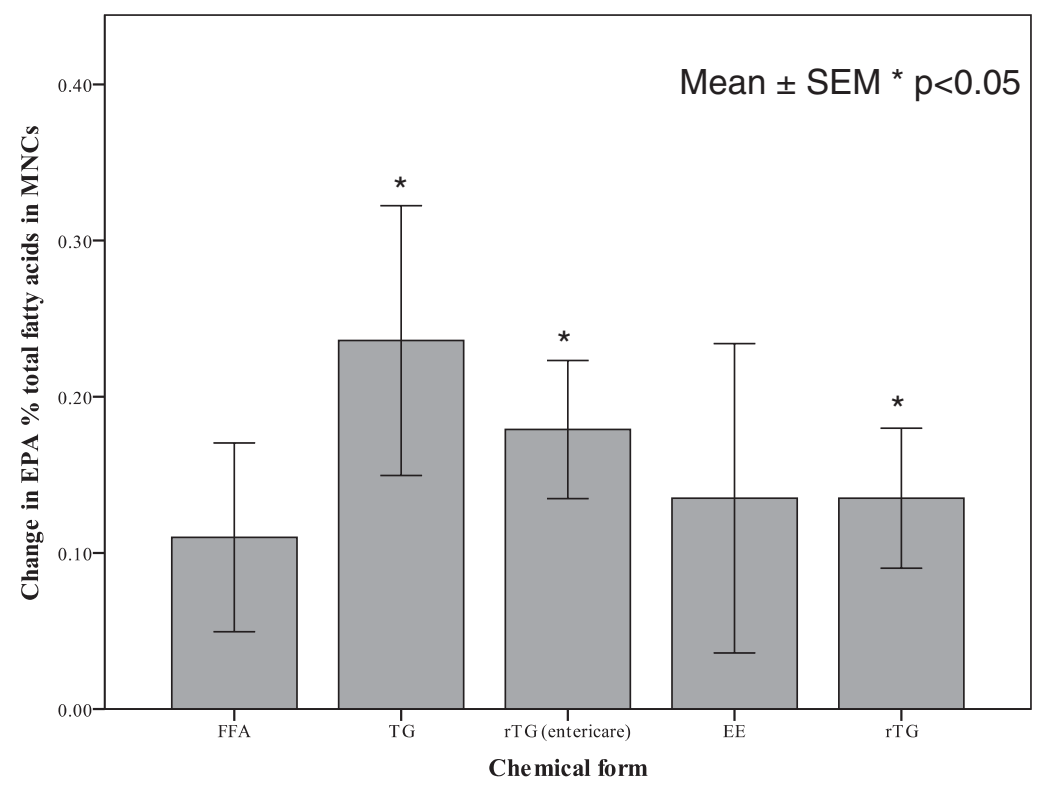

MNC EPA increased from baseline to 6 hours with all formulations of omega-3 fatty acids (see Figure). EPA was significantly higher at 6 hours than at baseline after ingesting TG, rTG and rTG (entericare) forms (all $p<0.05$ ). However, the change in MNC EPA over 6 hours did not differ among formulations. MNC DHA did not differ between baseline and 6 hours.

EPA is rapidly incorporated into immune cells in humans. The exact formation of the omega-3 supplement may influence this rapid incorporation, although the differences between formulations are small. Immune function could be modified by omega- 3 fatty acids more quickly than previously thought.

This research was possible thanks to an unrestricted research grant from Vifor Pharma.

1. Calder PC (2007) Prostaglandins Leuko Essent Fatty Acids 77, 327-335.

2. Calder PC (2008) Prostaglandins Leuko Essent Fatty Acids 79, 101-108.

3. Yaqoob P, Pala HS, Cortina-Borja M, Newsholme EA \& Calder PC (2000) Eur J Clin Invest 30, 260-274.

4. Faber J, Berkhout M, Vos AP et al. (2011) J Nutr 141, 964-970. 